Revista Brasileira de Agricultura Irrigada v.8, nº. 5, p.366 - 374, 2014

ISSN 1982-7679 (On-line)

Fortaleza, CE, INOVAGRI - http://www.inovagri.org.br

DOI: $10.7127 /$ rbai.v8n500232

Protocolo 232.14 - 21/03/2014 Aprovado em 03/09/2014

\title{
EVAPOTRANSPIRAÇÃO E DISPONIBILIDADE HÍDRICA EM FEIJOEIRO (Phaseolus vulgaris) SOB ESTRESSE HÍDRICO
}

\author{
João Alberto Fischer Filho ${ }^{1}$, João Luís Zocoler ${ }^{2}$, Nadia Maria Poloni ${ }^{3}$, Enes Furlani Júnior ${ }^{4}$
}

\begin{abstract}
RESUMO
Por ser cultivado durante o ano inteiro, o feijoeiro (Phaseolus vulgaris) fica exposto a diversas condições climáticas, tendo na deficiência hídrica um importante fator limitante à produção. Sendo assim, o uso da irrigação incrementa a produção, além de permitir a utilização de áreas antes impróprias devido aos regimes pluviométricos reduzidos ou irregulares. Este experimento, conduzido em casa de vegetação com vasos, teve o propósito de medir a evapotranspiração (ETr) e a água total disponível (AD) do feijoeiro sob estresse hídrico, relacionando-os com as fases fenológicas da cultura. Avaliou-se, também, entre os modelos de Veihmeyer \& Hendrickson, Thornthwaite \& Mather e Pierce qual representa melhor a relação entre evapotranspiração real e a potencial. O delineamento experimental foi inteiramente casualizado, com seis tratamentos (níveis de estresse hídrico) e quatro repetições, sendo um tratamento diferenciado pela vedação da superfície do solo. Com base nos resultados obtidos conclui-se que: i) a evapotranspiração no ciclo da cultura foi igual a 400, 378, 306, 251 e 198 mm, correspondente à água total disponível (AD) média de 70,4\%, $65,3 \%, 57,7 \%, 43,5 \%$ e $33,5 \%$, respectivamente; ii) o tratamento cuja cobertura do solo foi vedada a partir da fase fenológica V4 teve $\mathrm{AD}=68,3 \%$ resultando em evapotranspiração de $248 \mathrm{~mm}$; iii) o modelo de Thornthwaite \& Mather foi o que melhor se ajustou aos valores observados.
\end{abstract}

Palavras-chave: lâminas de irrigação, fases fenológicas do feijoeiro, deficiência hídrica.

\section{EVAPOTRANSPIRATION AND WATER AVAILABILITY AT BEAN (Phaseolus vulgaris) UNDER WATER STRESS}

\footnotetext{
ABSTRACT

Why grow some season, the bean be is exposed to various weather conditions, and water deficiency is an important limiting factor to production. Therefore, the use of irrigation increases the production and allows the use of areas previously unsuitable due to reduced or

${ }^{1}$ Mestrando em Agronomia/Ciência do Solo, Faculdade de Ciências Agrárias e Veterinárias, UNESP-Universidade Estadual Paulista, Campus de Jaboticabal. Email: joaofischer16@gmail.com

${ }^{2}$ Professor Adjunto, Faculdade de Engenharia, UNESP-Univ. Estadual Paulista, Campus de Ilha Solteira, Departamento de Fitossanidade, Engenharia Rural e Solos. Email: zocoler@agr.feis.unesp.br

${ }^{3}$ Mestranda em Agronomia/Sistemas de Produção, Faculdade de Engenharia, UNESP-Univ. Estadual Paulista, Campus de Ilha Solteira. Email: nadia_poloni@hotmail.com

${ }^{4}$ Professor Titular, Faculdade de Engenharia, UNESP-Univ. Estadual Paulista, Campus de Ilha Solteira, Departamento de Fitotecnia,

Tecnologia de Alimentos e Sócio Economia. Email: enes@agr.feis.unesp.br
} 
irregular rainfall regimes. This experiment, conducted on potted plants in greenhouse, intended to measure evapotranspiration (ETr) and total available water (AW) of the beans under water stress relating them with the phenological stages. It was also evaluated the best model that represents the relationship between real and potential evapotranspiration among Veihmeyer \& Hendrickson, Thornthwaite \& Mather and Pierce. The experiment was completely randomized with six treatments (levels of hydric stress) and four replications, and a differential treatment which the soil surface was sealing. It was concluded that: i) the evaporation cycle of the culture was equal to 400, 378, 306, 251 and $198 \mathrm{~mm}$, corresponding to total available water (AW) averaged $70.4 \%, 65.3 \%, 57.7 \%, 43.5 \%$ and $33.5 \%$, respectively; ii) the treatment which soil surface was sealed from the phenological stage V4 had AW $=68.3 \%$, resulting in evapotranspiration of $248 \mathrm{~mm}$; iii) Thornthwaite \& Mather was the best model that adjusted the observed values.

Keywords: depth irrigation, phenological stages of bean, hydric deficit.

\section{INTRODUÇÃO}

O feijoeiro comum (Phaseolus vulgaris, L.) é a leguminosa mais consumida no Brasil, sendo estimado em $16 \mathrm{~kg}$ o consumo anual per capita. Apresenta-se como uma cultura cultivada por produtores de pequeno a grande porte em praticamente todo território brasileiro assumindo grande importância socioeconômica. A cultura é considerada o principal alimento proteico no Brasil, uma vez que é alimento básico da classe populacional de receita mais baixa, tornando-se assim um alimento indispensável nas refeições da maioria dos brasileiros (ARF et al., 1996).

Por ser cultivado durante o ano inteiro o feijoeiro fica exposto a diversas condições climáticas. Entre os fatores climáticos, a deficiência hídrica é o mais importante (GUIMARÃES et al., 2006). Sendo assim, o uso da irrigação incrementa a produção agrícola por suprir a necessidade hídrica da cultura, também permite a utilização de áreas antes impróprias à produção agrícola devido regimes pluviométricos reduzidos ou irregulares. O principal objetivo da irrigação é proporcionar à cultura a quantidade de água necessária no momento certo, evitando assim redução da produtividade causada por estresse hídrico em fases críticas do seu ciclo
(BARROS, 2010), além de proporcionar que a cultura expresse todo o seu potencial produtivo. Santana et al. (2009) avaliaram o rendimento do feijoeiro comum-cultivar Talismã submetida a 5 níveis de reposição diária de água no solo para elevá-lo à capacidade de campo (40\%, 70\%, $100 \%, 130 \%$ e $160 \%$ ) e 3 níveis de suspensão da irrigação (0, 7 e 14 dias) após atingir o estádio R9 do ciclo cultural (modificação da cor das vagens e maturidade fisiológica das plantas). A análise de variância revelou efeito significativo das lâminas de reposição, das épocas de suspensão e da interação entre esses fatores. Houve aumento da produtividade até reposição de $100 \%$ com posterior queda. Também houve benefício da suspensão da irrigação na fase R9 exceto na reposição de $100 \%$, porém o efeito é similar, indicando que não se justifica a irrigação nesta fase.

Há muitas controvérsias quanto ao efeito da umidade do solo no decréscimo da relação ETr/ETm (ETr - evapotranspiração real, ETm evapotranspiração máxima ou potencial da cultura). Bernardo et al. (2006) citam três proposições. A primeira, de Veihmeyer \& Hendrickson, propõe que a evapotranspiração ocorre na razão potencial quando a umidade do 
solo está acima do ponto de murcha, caindo abruptamente quando se aproxima desse valor. A segunda, de Thornthwaite \& Mather, propõe um decréscimo linear da relação ETr/ETm com o decréscimo da umidade do solo. A terceira, de Pierce, propõe que a ETr se manterá acima de 90\% da ETm enquanto a umidade do solo estiver acima de, aproximadamente, um terço da água disponível, depois cairá mais rapidamente, na forma exponencial, até a umidade do solo no ponto de murcha.

Nesse contexto, ampliar conhecimentos em relação à demanda hídrica da planta nas diferentes fases fenológicas é importante para evitar a falta e/ou desperdício em períodos que a cultura requer menos ou mais água, agregando informações que possibilitem o aumento da produtividade e da qualidade dos grãos colhidos.

Este trabalho teve como objetivo medir a evapotranspiração (ETr) e a água total disponível (AD) do feijoeiro sob seis níveis de estresse hídrico relacionados às fases fenológicas da cultura. Além disso, avaliou-se entre os modelos de Veihmeyer \& Hendrickson, Thornthwaite \& Mather e Pierce qual representa melhor a relação entre a evapotranspiração real e a potencial.

\section{MATERIAL E MÉTODOS}

O trabalho foi realizado em casa de vegetação no Campus II da Faculdade de Engenharia/UNESP, Campus de Ilha Solteira (SP). Foi utilizado o feijão carioca cultivar BRSMG Majestoso, com semeadura realizada em 24 de junho de 2012.

O delineamento estatístico utilizado foi inteiramente casualizado, constituído por seis tratamentos e quatro repetições, descritos a seguir:

T0 - reposição de 100\% da transpiração, medida num período cuja depleção da água no solo foi entre 50 e $60 \%$ da Água Total Disponível (AD), tratamento com a superfície superior do vaso vedada para evitar a evaporação da água do solo;

T1 - tratamento no qual a planta praticamente não sofre estresse hídrico, ou seja, o solo permanece na capacidade de campo ou muito próximo a ela, havendo reposição diária de 100\% da evapotranspiração;

T2 - reposição de $100 \%$ da evapotranspiração, medida num período cuja depleção da água no solo foi entre 50 e $60 \%$ da AD. Nesse tratamento o estresse hídrico foi pequeno, porém maior que o tratamento 1 devido à reposição não necessariamente diária de água, mas corresponde a um período. Também diferiu do tratamento 0 , pois além da transpiração houve a evaporação da água do solo;

T3 - rega com reposição de 75\% em relação à T2;

T4 - rega com reposição de 50\% em relação à T2;

T5 - rega com reposição de $25 \%$ em relação à T2 ou pouco superior, se for necessário, para a planta não morrer.

Foram utilizados vasos de 10 litros com um Latossolo Vermelho escuro, sendo conduzida uma planta por vaso. O solo, antes da obtenção das amostras, foi peneirado (peneira grossa), homogeneizado e secado em terreiro até atingir a umidade residual ou próxima dela. Depois, o solo nos vasos foi saturado (umidade de saturação) e deixado drenar até a umidade da capacidade de campo, sendo coberta a parte superior dos mesmos para evitar a perda por evaporação. Ao se obterem pesagens sucessivas constantes, calculou-se a umidade à capacidade de campo. Amostras do solo foram enviadas aos 
laboratórios para análise de fertilidade, para análise granulométrica e para a obtenção da umidade do solo no ponto de murcha permanente.

Para realização das adubações foi seguida as recomendações de Raij et al. (1997). Para a adubação de plantio, foram aplicados $100 \mathrm{~kg} \mathrm{ha}^{-1}$ de sulfato de amônio, $34 \mathrm{~kg} \mathrm{ha}^{-1}$ de cloreto de potássio (KCl), $169 \mathrm{~kg} \mathrm{ha}^{-1}$ de superfosfato triplo, e $80 \mathrm{~kg} \mathrm{ha}^{-1}$ de superfosfato simples. Para as adubações nitrogenadas em cobertura, foram aplicados $250 \mathrm{~kg} \mathrm{ha}^{-1}$ de $\mathrm{N}$ na forma de sulfato de amônio, no início do estado fenológico V4 (terceira folha trifoliolada) e R8 (enchimento de grãos).

Após o plantio, os vasos foram mantidos em condições adequadas de umidade para o desenvolvimento das plantas até a fase fenológica V3 (emissão da primeira folha trifoliada). Após esta fase, os tratamentos foram diferenciados. As pesagens foram feitas diariamente, desde a semeadura ao fim do ciclo, sendo a diferença correspondente à evapotranspiração diária em kg por planta. Sempre que se fez necessária a reposição de água dos tratamentos, o volume foi aplicado com referência à diferença de pesagens e uso das relações entre os tratamentos. Assim, o cálculo para conversão da evapotranspiração total da cultura no ciclo, de kg por planta para lâmina em mm, foi conforme a Equação 1:

$$
\text { ETr total }(\mathrm{mm})=\frac{\operatorname{Pt} \times 10^{-3} \times 200000}{10000 \times 10^{-3}}
$$

Em que: Pt - peso total das ETr diárias (kg plan$\left.\mathrm{ta}^{-1}\right) ; 10^{-3}$ - volume de água por unidade de massa $\left(\mathrm{m}^{3} \mathrm{~kg}^{-1}\right) ; 200000$ - número de plantas por unidade de área (plantas ha-1); 10000 - conversão de área $\left(\mathrm{m}^{2} \mathrm{ha}^{-1}\right) ; 10^{-3}$ - conversão de extensão $\left(\mathrm{m} \mathrm{mm}^{-1}\right)$.

Para uso do modelo de Pierce a lâmina total de água (Lta, em mm) disponível e a lâmina atual de água (Laa, em mm) disponível no solo foram calculadas pelas equações 2 e 3, respectivamente:

$$
L t a=\frac{\left(\theta_{c c}-\theta_{p m p}\right) \times V s \times 10^{-3} \times 200000}{1 \times 10000 \times 10^{-3}}
$$

Em que: $\theta_{\text {co }}-$ umidade volumétrica na capacidade de campo $\left(\mathrm{m}^{3}\right.$ de água por $\mathrm{m}^{-3}$ de solo); $\theta_{\text {pmp }}$ - umidade volumétrica no ponto de murcha permanente ( $\mathrm{m}^{3}$ de água por $\mathrm{m}^{-3}$ de solo); Vs - volume de solo por vaso ( $\mathrm{L}$ vaso $\left.^{-1}\right) ; 10^{-3}$ _ conversão de volume $\left(\mathrm{m}^{3} \mathrm{~L}^{-1}\right) ; 200000$ - número de plantas por unidade de área (plantas ha-1); 1 número de plantas por vaso (planta vaso ${ }^{-1}$ );10000 - conversão de área $\left(\mathrm{m}^{2} \mathrm{ha}^{-1}\right) ; 10^{-3}$ - conversão de extensão $\left(\mathrm{m} \mathrm{mm}^{-1}\right)$.

$$
\text { Laa }=\operatorname{Lta} x \frac{A D(\%)}{100}
$$

Em que: $\mathrm{AD}$ - água disponível à base volumétrica $\left(\mathrm{m}^{3}\right.$ de água por $\mathrm{m}^{-3}$ de solo) multiplicada por 100 .

Procedeu-se a análise de variância e regressão da evapotranspiração do feijoeiro em função da água disponível no solo, que está relacionada aos tratamentos.

\section{RESULTADOS E DISCUSSÃO}

\section{Fases fenológicas do feijoeiro e a evapotranspiração}

Na Tabela 1, são apresentadas em relação a cada fase fenológica do feijoeiro, a duração em dias, data inicial e final (dia e mês), água total disponível média, evapotranspiração diária média e os valores totais e médios dos tratamentos propostos. Pode-se verificar que o ciclo do feijoeiro foi de 94 dias (da semeadura à colheita), e a fase R8 (enchimento de grãos) foi a mais longa, 22 dias. 


\section{EVAPOTRANSPIRAÇÃO E DISPONIBILIDADE HÍDRICA \\ EM FEIJOEIRO (Phaseolus vulgaris) SOB ESTRESSE HÍ́DRICO}

Tabela 1. Fases fenológicas do feijoeiro, duração em dias, data inicial e final (dia/mês do calendário 2012), evapotranspiração (ETr) e água total disponível (AD).

\begin{tabular}{|c|c|c|c|c|c|c|c|c|c|c|c|c|c|c|}
\hline \multirow{4}{*}{$\begin{array}{l}\mathscr{U} \\
\mathbb{J} \\
\widetilde{I}\end{array}$} & \multirow{4}{*}{$\stackrel{\mathscr{A}}{\mathscr{A}}$} & \multirow{4}{*}{$\begin{array}{l}\text { Dia } \\
\text { início }\end{array}$} & \multicolumn{12}{|c|}{ Tratamentos } \\
\hline & & & \multicolumn{2}{|c|}{ T0 } & \multicolumn{2}{|c|}{$\mathrm{T} 1$} & \multicolumn{2}{|c|}{$\mathrm{T} 2$} & \multicolumn{2}{|c|}{ T3 } & \multicolumn{2}{|c|}{$\mathrm{T} 4$} & \multicolumn{2}{|c|}{$\mathrm{T} 5$} \\
\hline & & & $\mathrm{AD}$ & $\mathrm{ETr}$ & $\mathrm{AD}$ & $\mathrm{ETr}$ & $\mathrm{AD}$ & $\mathrm{ETr}$ & $\mathrm{AD}$ & $\mathrm{ETr}$ & $\mathrm{AD}$ & $\mathrm{ETr}$ & $\mathrm{AD}$ & $\mathrm{ETr}$ \\
\hline & & & $\%$ & $\begin{array}{c}\mathrm{mm} / \\
\text { dia }\end{array}$ & $\%$ & $\begin{array}{c}\mathrm{mm} / \\
\mathrm{dia}\end{array}$ & $\%$ & $\begin{array}{c}\mathrm{mm} / \\
\text { dia }\end{array}$ & $\%$ & $\begin{array}{c}\mathrm{mm} / \\
\text { dia }\end{array}$ & $\%$ & $\begin{array}{c}\mathrm{mm} / \\
\text { dia }\end{array}$ & $\%$ & $\begin{array}{c}\mathrm{mm} / \\
\mathrm{dia}\end{array}$ \\
\hline \multirow[t]{2}{*}{ V0 } & 6 & & \multirow[t]{2}{*}{87} & \multirow[t]{2}{*}{2,1} & \multirow[t]{2}{*}{89} & \multirow[t]{2}{*}{1,9} & \multirow[t]{2}{*}{89} & \multirow[t]{2}{*}{1,9} & \multirow[t]{2}{*}{87} & \multirow[t]{2}{*}{2,1} & \multirow[t]{2}{*}{86} & \multirow[t]{2}{*}{2,1} & \multirow[t]{2}{*}{87} & \multirow[t]{2}{*}{2,1} \\
\hline & & 29/06 & & & & & & & & & & & & \\
\hline \multirow{2}{*}{ V1 } & 3 & 30/06 & \multirow{2}{*}{74} & \multirow{2}{*}{2,2} & \multirow{2}{*}{76} & \multirow{2}{*}{2,6} & \multirow{2}{*}{82} & \multirow{2}{*}{2,4} & 70 & 21 & 72 & 25 & 73 & 27 \\
\hline & & $02 / 07$ & & & & & & & & & & & & \\
\hline V2 & 5 & 03/07 & 70 & 23 & 66 & 28 & 73 & 26 & 76 & 21 & 60 & 31 & 57 & 33 \\
\hline & & $10 / 07$ & & & & & & & & & & & & \\
\hline V3 & 9 & 08/07 & 68 & 22 & 63 & 23 & 65 & 23 & 72 & 22 & 59 & 25 & 56 & 26 \\
\hline & & $16 / 07$ & & & & & & & & & & & & \\
\hline V4 & 15 & $17 / 07$ & 65 & 25 & 60 & 47 & 60 & 43 & 56 & 39 & 46 & 32 & 34 & 27 \\
\hline & & $31 / 07$ & & & & & & & & & & & & \\
\hline $\mathrm{DF}$ & 10 & 01/08 & 62 & 4 & 74 & & & & & & & & & 18 \\
\hline R5 & 10 & 10/08 & 63 & 4,4 & 74 & 6,6 & 63 & 5,6 & 42 & 4,8 & 22 & 3,3 & 9 & 1,8 \\
\hline R6 & 5 & $11 / 08$ & 64 & 43 & 70 & 51 & 60 & 56 & 34 & 4.4 & 15 & 29 & 9 & 18 \\
\hline & & $15 / 08$ & & & & & & & & & & & & \\
\hline R7 & 0 & $16 / 08$ & ca & 30 & Co & 50 & co & 57 & 48 & 20 & 33 & 7 & 25 & 2 \\
\hline & & $24 / 08$ & & & & & & & & & & & & \\
\hline R8 & 22 & $25 / 08$ & 67 & 2,1 & 72 & 4,4 & 61 & 4,3 & 58 & 3,1 & 42 & 2,5 & 25 & 1,7 \\
\hline & & $15 / 09$ & & & & & & & & & & & & \\
\hline R9 & 19 & $16 / 09$ & 68 & 1,3 & 69 & 4,2 & 59 & 3,0 & 44 & 2,4 & 27 & 1,8 & 15 & 1,4 \\
\hline & & $25 / 09$ & & & & & & & & & & & & \\
\hline
\end{tabular}

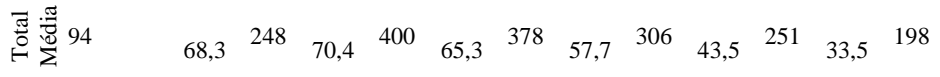

V0: germinação; V1: emergência; V2: folhas primárias; V3: primeira folha trifoliolada; V4: terceira folha trifoliolada; R5: pré-floração; R6: floração; R7: formação de vagens; R8: enchimento de grãos; R9: maturação.

Verificou-se significância da regressão linear da ETr em função da AD ao nível de 5\%, conforme pode ser observada na Tabela 2, com coeficiente de ajuste de 98,15\%, ou seja, a redução da evapotranspiração apresenta linearidade com a redução da água disponível no solo, que está diretamente relacionada aos tratamentos de 1 a 5. Em relação aos tratamentos, verificouse que o tratamento 1 (T1) apresentou a maior evapotranspiração no ciclo da cultura, $400 \mathrm{~mm}$, seguido do tratamento dois (T2), com $378 \mathrm{~mm}$, do tratamento 3, com $306 \mathrm{~mm}$, do tratamento 4, com 251 mm, e do tratamento 5, com 198 mm. A evapotranspiração total decrescente do tratamento 1 ao 5 está relacionada ao decréscimo da água total disponível (AD), em média, 70,4\%, $65,3 \%, 57,7 \%, 43,5 \%$ e $33,5 \%$, respectivamente. Em outras palavras, ocorreu um aumento do módulo do potencial mátrico do solo, tornando o fluxo da água no sistema solo-planta-atmosfera menor, o que proporcionou uma concentração de vapor d’água no mesófilo da folha menor que a saturação, e consequentemente, redução na taxa de transpiração. Além disso, os tratamentos que possuem maiores $\mathrm{AD}$ também sofreram maiores perdas por evaporação devido à concentração de umidade nas proximidades da superfície do solo.

Tabela 2. Síntese da análise de variância e regressão da evapotranspiração no ciclo do feijoeiro (ETr, em mm) em função da água total disponível no solo (AD, em \%).

\begin{tabular}{ccccc} 
Modelo & Equação & NMS & $\mathrm{R}^{2}(\%)$ & $\begin{array}{c}\mathrm{CV} \\
(\%)\end{array}$ \\
\hline Linear & $\mathrm{ETr}=11,86+5,451 \mathrm{AD}$ & 0,028 & 98,15 & 4,33 \\
Quadrático & $\begin{array}{c}\mathrm{ETr}=130,8+0,515 \mathrm{AD} \\
+0,0475 \mathrm{AD}^{2}\end{array}$ & 0,055 & 98,82 & 4,33 \\
& & & & \\
\hline
\end{tabular}

No tratamento 0 , no qual a superfície do solo estava vedada após a fase V3, a evaporação foi praticamente nula. Ocorreu apenas a transpiração da planta, processo consumidor de energia, que tem como função moderar a temperatura da folha, sujeita à radiação solar. Nesse tratamento a evapotranspiração total foi apenas 238 mm, apresentando AD em média 68,3\%, ou seja, para disponibilidade hídrica semelhante aos tratamentos 1 e 2, a evapotranspiração foi apenas 62 e 66\%, respectivamente. Andrade et al. 
(2002), ao estudar o consumo relativo de água do feijoeiro no plantio direto em função da porcentagem de cobertura morta do solo, verificou que a evapotranspiração apresentou valor menor à medida que se aumentou a porcentagem de cobertura do solo pela palhada, portanto, ocorre um aumento da eficiência no uso da água com a redução do consumo.

A Figura 1 ilustra a tendência dos valores da evapotranspiração (ETr) diária média em relação às fases fenológicas da cultura.

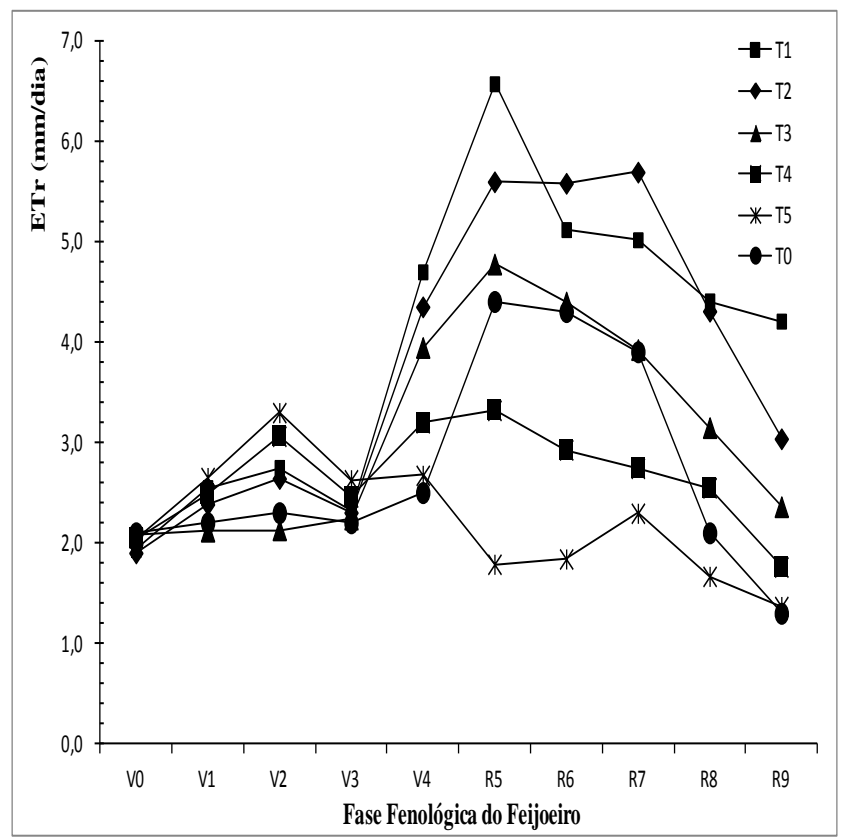

Figura 1. ETr diária média de cada tratamento em função das fases fenológicas do feijoeiro.

Com base na Tabela 1 e Figura 1 é possível verificar que a fase R5 (pré-floração), foi a que apresentou a maior evapotranspiração diária, exceto os tratamentos 5 e 2 . No tratamento 5 ocorreu maior estresse hídrico, em média 9\% da $\mathrm{AD}$, valor que ficou abaixo da média geral do próprio tratamento, de 33,5\%. Em relação ao tratamento 2, observou-se que o pico de evapotranspiração diária ocorreu na fase R7 (formação de vagens), valor atribuído à variação do acaso, embora as fases R6 (floração) e R7 também apresentaram elevada evapotranspiração. Kelling (1995), ao estudar o efeito da disponibilidade de água no solo sobre os componentes do balanço hídrico, verificou que os subperíodos de maior exigência hídrica foram a R5 (pré-floração), R6 (floração) e R7 (formação de vagens), indicando que um menor estresse nestes períodos evitaria problemas como o abortamento de flores, queda das estruturas florais e a queda das vagens pequenas, em consequência da redução do metabolismo da planta com o fechamento dos estômatos.

As fases V0 (germinação) e a R9 (maturação) foram as que apresentaram os menores valores de evapotranspiração diária. No primeiro caso, devido à planta ainda não ter emitido as folhas a transpiração foi praticamente nula, só ocorreu evaporação da superfície do solo. No segundo caso, fase R9, a redução da evapotranspiração diária ocorreu devido à redução das atividades metabólicas da planta, assim as folhas envelheceram até a morte da parte aérea.

A evapotranspiração no ciclo das culturas em geral segue a tendência de aumentar à medida que a planta se desenvolve, alcançando um máximo na época da floração e o decréscimo da evapotranspiração inicia-se com o início da maturação da planta. A evapotranspiração diária da fase V3 (primeira folha trifoliada) foi inferior àquela da fase V2 (folhas primárias) em praticamente todos os tratamentos, exceto o tratamento 3. Isso ocorreu, provavelmente, porque a fase V3 coincidiu com o período de queda da temperatura do ar entre os dias 8 e 16 de julho de 2012. Mesmo considerando que as plantas estavam em ambiente protegido, a temperatura interna segue a tendência da externa. O comportamento diferente do tratamento 3, aparentemente, é atribuído ao seu maior teor médio de $\mathrm{AD}$ em relação aos demais (Tabela 1), lembrando que até esta fase fenológica não havia diferenciação dos tratamentos. 
Conforme a Figura 1, é possível verificar, também, que o tratamento 0 apresentou transpiração superior à evapotranspiração dos tratamentos 4 e 5 nas fases fenológicas R5, R6 e R7, mostrando que tanto a evaporação como a transpiração nesses tratamentos foram baixas devido à restrição hídrica. No caso da evaporação, formou-se uma camada superficial seca no solo que reduziu a condutividade hidráulica; enquanto no caso da transpiração o estresse hídrico causou o fechamento estomático.

Se comparado com o tratamento 2, que apresentou equivalência em relação à proposta de reposição de $100 \%$ da evapotranspiração, medida num período cuja depleção da água no solo estava entre 50 e $60 \%$ da $\mathrm{AD}$, o tratamento 0 apresentou, a partir da fase V4, menor transpiração em todas as fases fenológicas da cultura. Isso foi devido ao efeito combinado evaporaçãotranspiração do tratamento 2 em relação a somente a transpiração do tratamento 0 .

\section{Avaliação dos modelos Veihmeyer \& Hendrickson, Thornthwaite \& Mather e Pierce}

Para se avaliar o modelo que melhor representa o decréscimo da relação ETr/ETp em função da $\mathrm{AD}$, uma exigência fundamental é o conhecimento da evapotranspiração máxima ou potencial. O tratamento 1 tinha o propósito de medir a evapotranspiração potencial do feijoeiro, mantendo a umidade do solo próxima à capacidade de campo, ou seja, $\mathrm{AD} \cong 100 \%$. Todavia, na prática, a reposição diária não foi suficiente para tal, o que foi atribuído à temperatura elevada e umidade relativa do ar baixa durante o ciclo da cultura, cujos valores medidos no lado externo da estufa foram, respectivamente, 23,7 ${ }^{\circ} \mathrm{C}$ e $59,7 \%$, respectivamente. Com isso, no tratamento 1 , conseguiu-se ter um valor médio de $\mathrm{AD}$ de $70,4 \%$ e não $100 \%$ que se pretendia. Sendo assim, a evapotranspiração potencial do feijoeiro foi estimada com base na regressão dos valores da evapotranspiração real versus a água disponível (AD), cuja síntese da análise de variância e regressão é mostrada na Tabela 2 . Como a regressão linear foi a que teve significância para o limite de 5\%, substituindo-se o valor de 100 na equação, a evapotranspiração máxima ou potencial estimada para o ciclo do feijoeiro foi de $557 \mathrm{~mm}$, valor este considerado nos ajustes dos modelos propostos, mostrados na Figura 2.

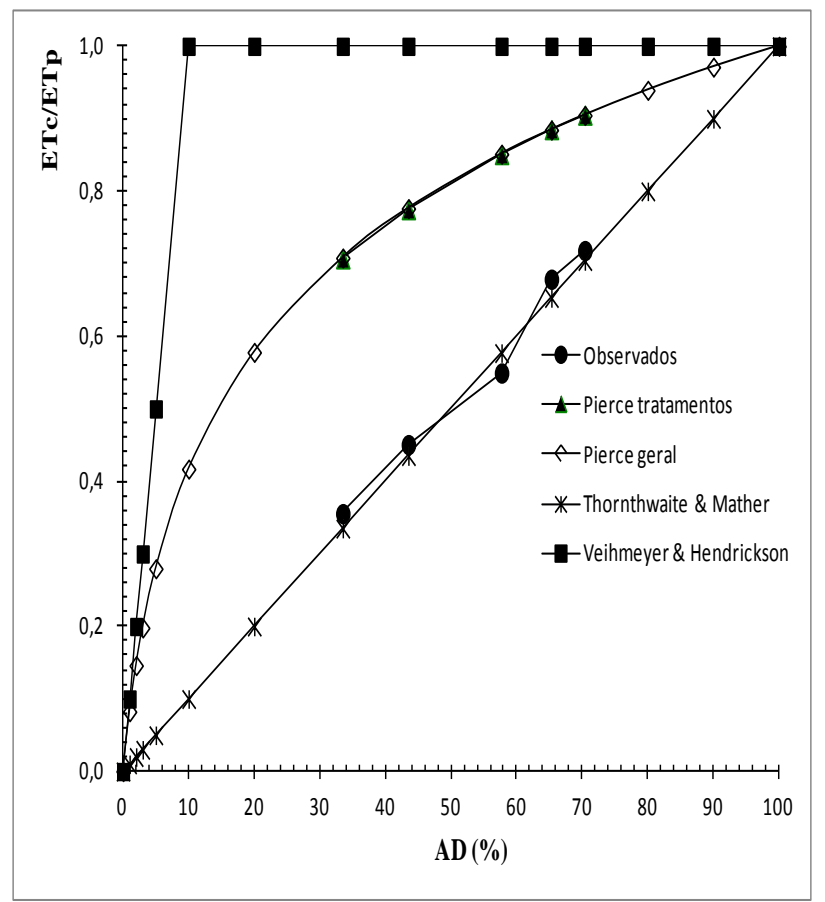

Figura 2. Relação entre evapotranspiração real/potencial (ETr/ETp) em função da água total disponível no solo (AD).

Para uso do modelo de Pierce aplicado separadamente para os tratamentos, a lâmina total de água (Lta) disponível e a lâmina atual de água (Laa) disponível foram calculadas pelas Equações 2 e 3, sendo obtidos os seguintes valores médios para cada tratamento:

- Tratamento 1: Lta $=34,6 \mathrm{~mm}$ e Laa $=24,3 \mathrm{~mm}$; - Tratamento 2: Lta = 34,9 mm e Laa = 22,8 mm; 
- Tratamento 3: Lta = 34,3 mm e Laa = 19,7 mm;

- Tratamento 4: Lta $=33,3 \mathrm{~mm}$ e Laa $=14,5 \mathrm{~mm}$;

- Tratamento 5: Lta $=33,4 \mathrm{~mm}$ e Laa $=11,2 \mathrm{~mm}$.

No caso do modelo de Pierce geral, a lâmina total de água (Lta) disponível foi utilizada a média geral, 34,1 mm e lâmina atual de água foi estimada pela Equação 3. Salienta-se que o tratamento 0 , por apresentar a particularidade de vedação da superfície do solo, não fez parte da avaliação dos modelos.

Conforme pode ser visto na Figura 2, o modelo de Thornthwaite \& Mather foi o que mais se assemelhou aos valores observados. Desta maneira, observou-se um decréscimo linear da relação ETr/ETp com o decréscimo da umidade do solo. $\mathrm{O}$ resultado obtido não corroborou o relato de Bernardo et al. (2006) no qual a maioria dos autores acreditam que a ETr é igual à ETp durante algum tempo, decrescendo segundo uma forma exponencial, isto é, de acordo com modelo de Pierce. O modelo de Pierce aplicado aos tratamentos (Pierce tratamentos na Figura 2) estimaria ETc/ETp maiores que os observados.

\section{CONCLUSÕES}

De acordo com os objetivos do trabalho pode-se concluir que:

- A redução da evapotranspiração apresenta linearidade com a redução da água disponível no solo, caindo de $400 \mathrm{~mm}$ quando a $\mathrm{AD}$ era $70,4 \%$ para $198 \mathrm{~mm}$ quando a $\mathrm{AD}$ era $33,5 \%$;

- Com isso, entre os modelos avaliados, o de Thornthwaite \& Mather foi o que apresentou as melhores estimativas de evapotranspiração em relação aos valores observados;

- No tratamento que teve a cobertura do solo vedada a partir da fase fenológica V4 a evapotranspiração foi 62 e $66 \%$ da evapo- transpiração observada nos tratamentos com similar disponibilidade hídrica, porém sem cobertura do solo.

\section{REFERÊNCIAS BIBLIOGRÁFICAS}

ANDRADE, R. S.; MOREIRA, J. A. A.; STONE, L. F.; CARVALHO, J. A. Consumo relativo de água do feijoeiro no plantio direto em função da porcentagem de cobertura morta do solo. Revista Brasileira de Engenharia Agrícola e Ambiental, v.6, n.1, p.35-38, 2002.

ARF, O.; SÁ, M. E.; OKITA, C. S.; TIBA, M. A.; GUERREIRO NETO, G.; OGASSAWARA, F. Y. Efeito de diferentes espaçamentos e densidade de semeadura sobre o desenvolvimento do feijoeiro (Phaseolus vulgaris L.). Pesquisa Agropecuária Brasileira, v. 31, n. 9, p. 629 - 634, 1996.

BERNARDO, S.; SOARES, A. A.; MANTOVANI, E. C. Manual de irrigação. 8. ed. Viçosa: UFV, 2006. 625p.

BARROS, F. C. Uso de mangueiras de polietileno linear de baixa densidade na irrigação da cultura do feijoeiro. 2010. TCC (Graduação em Agronomia) - Faculdade de Engenharia de Ilha Solteira, Universidade Estadual Paulista, Ilha Solteira, 2010.

GUIMARÃES, C. M.; STONE, L. F.; BRUNINI, O. Adaptação do feijoeiro comum (Phaseolus vulgaris L.) à seca. Revista Brasileira de Engenharia Agrícola e Ambiental, v.10, n.1, p.70-75, 2006.

KELLING, C. R. S. Efeito da disponibilidade de água no solo sobre os componentes do balanço hídrico e o rendimento do feijoeiro. 1995. 91f. Dissertação (Mestrando em Engenharia Agrícola) - Universidade Federal de Santa Maria, Santa Maria, 1995.

RAIJ, B. van; CANTARELLA, H.; QUAGGIO, J. A.; FURLANI, A. M. C. Recomendações de 
EVAPOTRANSPIRAÇÃO E DISPONIBILIDADE HÍDRICA

EM FEIJOEIRO (Phaseolus vulgaris) SOB ESTRESSE HÍDRICO

adubação e calagem para o estado de São

Paulo. Campinas: Instituto Agronômico/ Fundação IAC, 1997. 285 p.

SANTANA, M. J; CARVALHO, J. A.; ANDRADE, M. J. B.; GERVÁSIO, G. G.;
BRAGA, J. C.; LEPRI, E. B. Viabilidade técnica e econômica da aplicação de água na cultura do feijoeiro comum (Phaseolus vulgaris L.). Ciência e Agrotecnologia, v. 33, n. 2, p. 532538, 2009. 